\title{
Erratum to: Introduction
}

\author{
Erratum to: \\ M. Orsag et al., Aerial Manipulation, \\ Advances in Industrial Control, \\ https://doi.org/10.1007/978-3-319-61022-1_1
}

Chapter 1 in the original version of this book was inadvertently published without the name of Prof. Anibal Ollero as the author of this introductory chapter.

The updated online version of this chapter can be found at https://doi.org/10.1007/978-3-31961022-1_1 